\title{
Figure of merit comparison of PP-based electret and PVDF-based piezoelectric polymer energy harvesters
}

\author{
M. Mrlík ${ }^{1,2}$, S. Leadenham ${ }^{3}$, M. A. AlMaadeed ${ }^{1}$, and A. Erturk ${ }^{3, *}$ \\ ${ }^{1}$ Center for Advanced Materials, Qatar University, P. O. BOX 2713, Doha, Qatar \\ ${ }^{2}$ Centre of Polymer Systems, Tomas Bata University in Zlin, Trida T. Bati 5678, 76001 Zlin, Czech Republic \\ ${ }^{3}$ G. W. Woodruff School of Mechanical Engineering, Georgia Institute of Technology, Atlanta, Georgia, 30332,
} USA

\begin{abstract}
The harvesting of mechanical strain and kinetic energy has received great attention over the past two decades in order to power wireless electronic components such as those used in passive and active monitoring applications. Piezoelectric ceramics, such as PZT (lead zirconate titanate), constitute the most commonly used electromechanical interface in vibration energy harvesters. However, there are applications in which piezoelectric ceramics cannot be used due to their low allowable curvature and brittle nature. Soft polymer PVDF (polyvinylidene fluoride) is arguably the most popular non-ceramic soft piezoelectric energy harvester material for such scenarios. Another type of polymer that has received less attention is PP (polypropylene) for electret-based energy harvesting using the thickness mode (33mode). This work presents figure of merit comparison of PP versus PVDF for off-resonant energy harvesting in thickness mode operation, revealing substantial advantage of PP over PVDF. For thicknessmode energy harvesting scenarios (e.g. dynamic compression) at reasonable ambient vibration frequencies, the figure of merit for the maximum power output is proportional to the square of the effective piezoelectric strain constant divided by the effective permittivity constant. Under optimal conditions and for the same volume, it is shown that PP can generate more than two orders of magnitude larger electrical power as compared to PVDF due to the larger effective piezoelectric strain constant and lower permittivity of the former.
\end{abstract}

Keywords: Energy harvesting, polymers, polypropylene, polyvinylidene fluoride, electret, piezoelectric.

\section{INTRODUCTION}

Vibration-based energy harvesting using piezoelectric materials has been heavily researched in the past two decades [1-5]. The goal in this research field is to enable self-powered wireless electronic components by converting ambient vibrations into electricity. The broad range of potential applications spans from structural health monitoring sensors to biomedical implants. Piezoelectric ceramics, e.g. PZT5A and PZT-5H (PZT: lead zirconate titanate), constitute the most commonly used electromechanical interface in vibration energy harvesters $[2,3,5,6]$. There are applications (e.g. wearable electronics) in which piezoelectric ceramics cannot be used due to their low allowable curvature and brittle nature. Polymer PVDF (polyvinylidene fluoride) is the most commonly used non-ceramic soft piezoelectric energy harvester material [7-10] that offers mechanical compliance at the cost of reduced electromechanical coupling. Another type of polymer that has received less attention is PP

\footnotetext{
*alper.erturk@me.gatech.edu; phone 1404385 1394; fax 1404894 8496; www.ssdsl.gatech.edu
} 
(polypropylene) for electret-based energy harvesting [11] using the effective thickness mode (33-mode). The formation of PP films involves a process in which biaxial stretching results in lens-like voids in the material due to initially compound small particles such as minerals (which act as nuclei for void creation) prior to corona discharging $[12,13]$. There has been growing interest $[11,14-16]$ in the use of PP films for energy harvesting as an alternative to PVDF [8-10] and other soft materials such as dielectric elastomers $[17,18]$ and ionic polymer-metal composites $[19,20]$.

In this paper we perform a detailed comparison of electret PP and piezoelectric PVDF polymer materials for their energy harvesting capabilities. The focus is placed on the typical off-resonant frequency range scenarios of such soft polymer films. It is aimed to identify and compare the energy harvesting figure of merit for the effective 33-mode implementation of PP and PVDF polymers. In the following, first the experimental test samples are introduced with a focus on their microscopic structures and chemical compositions. Then, a lumped-parameter electromechanical model is described for the firstorder base excitation problem of a polymer film loaded by a seismic mass and connected to a resistive electrical load. This model is first used for the effective piezoelectric constant identification. Capacitance properties of the samples are also identified. The model is then extended to optimal energy harvesting condition of each sample for a fair comparison and a figure of merit is identified. Finally the PP and PVDF samples are compared in terms of their power generation capabilities under identical excitation and volume.

\section{TEST SAMPLES: PP ELECTRET AND PVDF PIEZOELECTRIC POLYMERS}

The PP samples were acquired from the Emfit Ltd. (Finland) while the PVDF samples were manufactured by the Measurement Specialties, Inc. (US). In order to investigate their internal microscopic structures and chemical compositions, these polymer samples were cut in liquid nitrogen and their crosssections were obtained using Scanning Electron Microscopy (SEM) as shown in Fig. 1. In the uncoated PP sample, the cross-section clearly shows the porous structure of the polymer with lens-like voids, and the total thickness was estimated to be $80 \mu \mathrm{m}$. Each void includes a small spherical particle of calcium carbonate, which is mixed to the polymer melt as a nucleation agent for void creation. The PP sample coated with aluminum has lower thickness (which was estimated to be $70 \mu \mathrm{m}$ ) with nearly same size and shape of the void. However, due to the additional coating, the sample probably had to be pressed and thus the dimensions are slightly different as compared to its uncoated PP counterpart. The last sample is the PVDF which does not have any porous structures and the thickness of the sample was estimated to be 110 $\mu \mathrm{m}$. These thicknesses are in agreement with the respective manufacturers' data sheets.

(a)

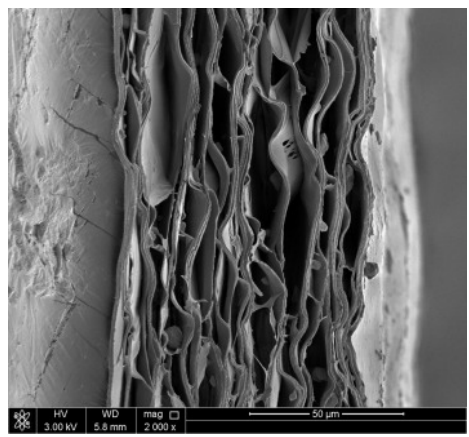

(b)

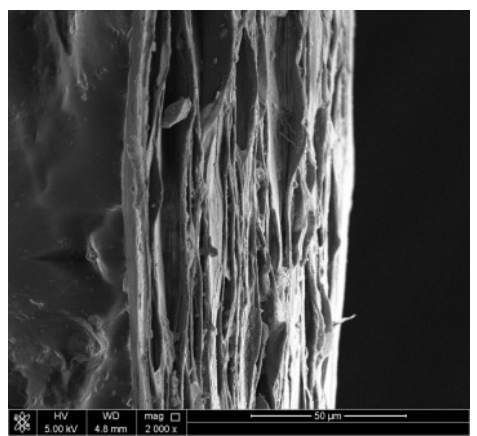

(c)

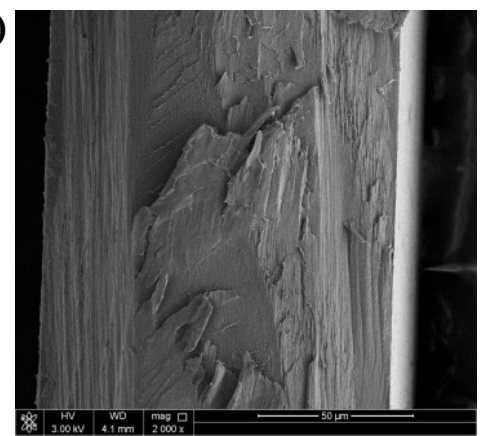

Figure 1. SEM cross-section images of (a) uncoated PP (total thickness $\sim 80 \mu \mathrm{m}$ ), (b) coated PP (total thickness $\sim 70 \mu \mathrm{m}$ ), and (c) PVDF (total thickness $\sim 110 \mu \mathrm{m}$ ) samples. 
As a part of the SEM analysis, results of Energy Dispersive Spectroscopy (EDS) show the chemical composition of the polymer electret samples in Fig. 2. As can be seen in Fig. 2a, the PP samples include mostly carbon and small amount of calcium if the investigated portion is polymer void. If the electron beam is focused on the aforementioned spheres, it is revealed that they consist of calcium, carbon and oxygen, which clearly indicates the calcium carbonate nature (Fig. 2b). In case of PVDF, fluorine and carbon amounts are comparable, which is consistent with what is expected (Fig. 2c). The amounts of the elements that are present in various polymer electret samples are also given in Table 1.
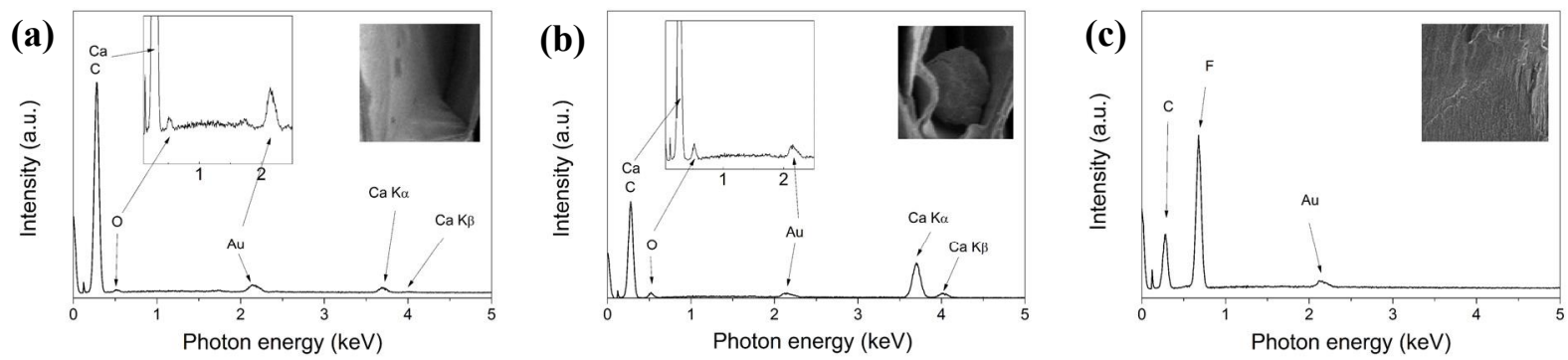

Figure 2. EDS spectra of the PP and PVDF samples: (a) pure PP electret void; (b) calcium carbonate sphere in the PP sample; and (c) PVDF sample. In the PP sample cases the left inset is the magnified spectra and the right inset is the investigated region.

Table 1. Amounts of the elements present in PP (along with calcium carbonate) and PVDF samples.

\begin{tabular}{ccccccc}
\hline Sample names & \multicolumn{2}{c}{ PP } & \multicolumn{2}{c}{ Calcium carbonate } & \multicolumn{2}{c}{ PVDF } \\
\hline Elements & wt. \% & at. \% & wt. \% & at. \% & wt. \% & at. \% \\
\hline C & 98.5 & 99.5 & 73.0 & 84.0 & 42.4 & 53.8 \\
F & - & - & - & - & 57.6 & 46.2 \\
Ca & 1.5 & 0.5 & 14.1 & 4.9 & - & - \\
O & - & - & 12.9 & 11. & - & - \\
\hline
\end{tabular}

\section{ELECTROMECHANICAL MODELING AND PARAMETER IDENTIFICATION}

In order to identify the electromechanical coupling constants of PP and PVDF samples and to quantify their relative energy harvesting performance results, a series of dynamic experiments were conducted. As shown schematically in Fig. 3a, the setup consists of the PP or PVDF sample sandwiched between a conductive seismic mass on top and a conductive foil layer below. The seismic mass and the foil layer form the electrodes with wires connecting them to the shunt resistance box. Beneath the foil layer is an insulating layer that isolates the sample from the metal shaker platform, which oscillates harmonically in the vertical direction. An accelerometer is used to measure the motion of the shaker table. The overall experimental setup picture with a close-up view is displayed in Fig. $3 \mathrm{~b}$.

The lumped-parameter model (Fig. 3c) of the 33-mode (thickness mode) off-resonant energy harvester configuration can be given by the following first-order ordinary differential equation:

$$
C \dot{v}(t)+\frac{v(t)}{R}=d_{33} \dot{F}(t)
$$


where $F$ is the force on the device in the poling direction, $C$ is the static capacitance of the device, $R$ is the load resistance, $v$ is the voltage across the load resistance, $d_{33}$ is the equivalent piezoelectric strain constant (due to piezoelectric or electret effects), and an over-dot represents the derivative with respect to time. This first-order model is applicable when the dominant frequency of the applied force is well below the fundamental resonance frequency of the system formed by the elastic PP or PVDF sample and the seismic mass (in agreement with the experiments of interest in this work, i.e. $\sim 100 \mathrm{~Hz}$ ).

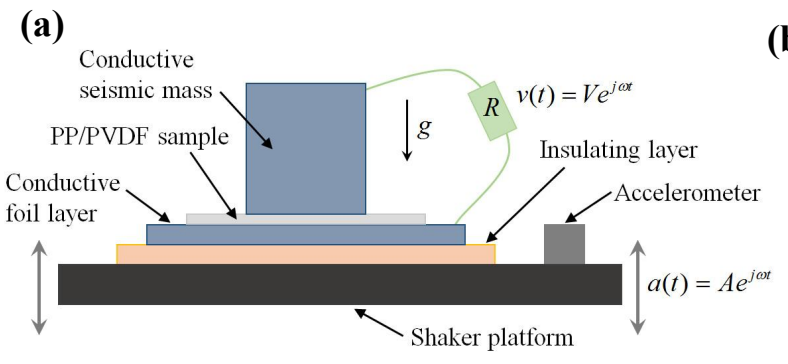

(b)

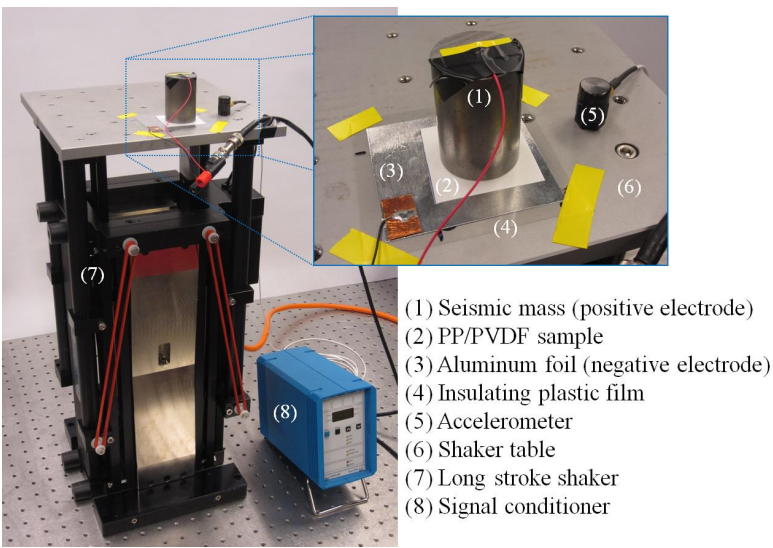

Figure 3. Experimental setup details: (a) Schematic and (b) pictures of the setup components; and (c) lumped-parameter electrical circuit representation. The electrical output of the PP/PVDF sample is connected to a resistive electrical load for parameter identification and energy harvesting performance quantification.

In the experimental setup shown in Figs. 3a-b, the dynamic forcing is provided by the shaker motion and the seismic mass. That is,

$$
F(t)=m a(t)
$$

where $m$ is the mass of the seismic mass, and $a(t)$ is the vertical acceleration of the shaker table. Assuming harmonic excitation at the radial frequency $\omega$ and linear system behavior, one can find the following frequency response function (FRF) which relates the complex voltage amplitude $(V)$ across the load resistance to the base acceleration input $(A)$ :

$$
\frac{V}{A}=\frac{j R m d_{33} \omega}{1+j R C \omega}
$$

This FRF could be easily rearranged to give the harvested power in such base acceleration experiments as discussed in the next section. However, our first intention is parameter identification. To extract the values of $d_{33}$ for PP and PVDF samples, harmonic base acceleration frequency sweep tests were conducted with various load resistance values. Mass values were chosen to isolate the frequencies of interest for the tests from spurious vibration modes in the shaker and shaker table. All masses have the same contact area of $9.56 \mathrm{~cm}^{2}$. Frequencies chosen were low $(R C \omega<<1)$, yielding experimental frequency response functions that are approximately linear, i.e.

$$
\left|\frac{V}{A}\right| \approx \omega m d_{33} R
$$

Experimental frequency response functions for the three samples are shown in Fig. 4. The identified effective thickness-mode piezoelectric strain constant $\left(d_{33}\right)$ values are summarized in Table 2. 
The electromechanical coupling values for the PVDF sample is in the expected range found in literature. The coated PP results in substantially larger $d_{33}$ value. Note that, expectedly, the results are consistent for different resistance values.
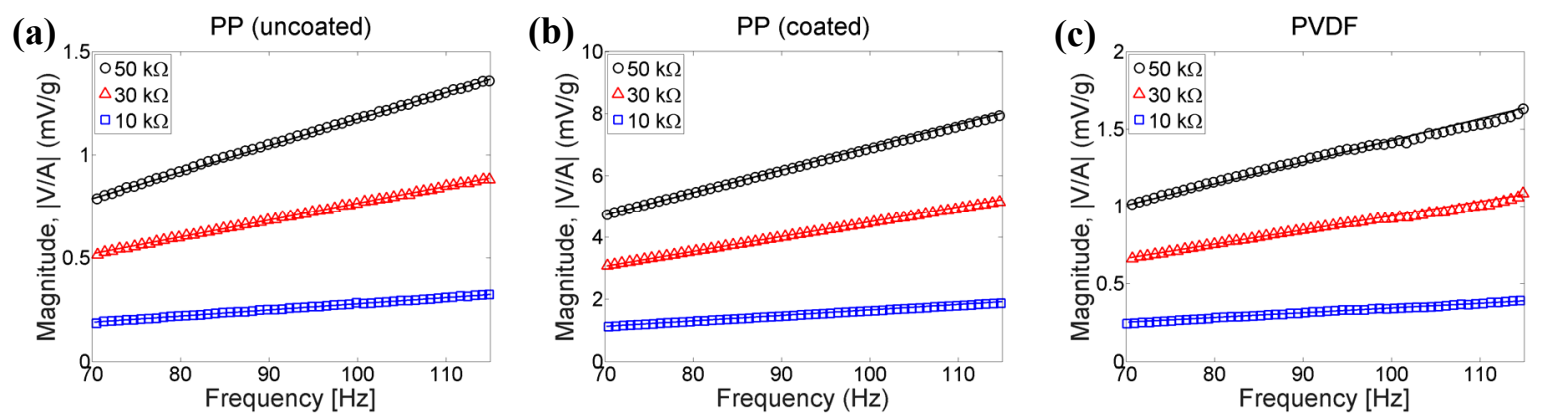

Figure 4. Voltage per base acceleration FRF magnitudes of the test samples for a set of resistive electrical loads: (a) uncoated PP; (b) coated PP; and (c) PVDF. Seismic masses used are 284g, 377g, and 377g, respectively. Load resistances of $10 \mathrm{k} \Omega, 30 \mathrm{k} \Omega$, and $50 \mathrm{k} \Omega$ are shown by blue squares, red triangles, and black circles, respectively. Model simulations are shown as solid lines of the same color.

Table 2. Summary of the identified $d_{33}$ values for the uncoated PP, coated PP, and PVDF.

\begin{tabular}{lcccc}
\hline & $10 \mathrm{k} \Omega$ & $30 \mathrm{k} \Omega$ & $50 \mathrm{k} \Omega$ & Average \\
\hline PP (uncoated) & $13.5 \mathrm{pC} / \mathrm{N}$ & $13.6 \mathrm{pC} / \mathrm{N}$ & $13.7 \mathrm{pC} / \mathrm{N}$ & $13.6 \mathrm{pC} / \mathrm{N}$ \\
PP (coated) & $76.3 \mathrm{pC} / \mathrm{N}$ & $76.7 \mathrm{pC} / \mathrm{N}$ & $77.0 \mathrm{pC} / \mathrm{N}$ & $76.7 \mathrm{pC} / \mathrm{N}$ \\
PVDF & $19.5 \mathrm{pC} / \mathrm{N}$ & $19.0 \mathrm{pC} / \mathrm{N}$ & $18.8 \mathrm{pC} / \mathrm{N}$ & $19.1 \mathrm{pC} / \mathrm{N}$
\end{tabular}

To complete the model identification process, the static capacitance of each sample was measured with various preloads caused by different seismic masses. The measured capacitance results are summarized in Table 3. Note that there is a clear trend of increasing capacitance with increasing static load with both PP samples. This is likely due to the compressive loading deforming the voids in the PP samples. The PVDF sample, which has a higher effective Young's modulus and no internal voids, is much more insensitive to changes in compressive preload. PP has a typical relative permittivity value of between 2.2 and 2.4. Using the measured capacitance values of the coated and uncoated PP samples, the estimates for effective relative permittivity are approximately unity. These low values may be caused by the internal structure of the film. The voids act like individual capacitors in series, reducing the effective capacitance of the bulk film.

Table 3. Summary of the identified capacitance values for the uncoated PP, coated PP, and PVDF.

\begin{tabular}{lcccccc}
\hline & $94 \mathrm{~g}$ & $190 \mathrm{~g}$ & $284 \mathrm{~g}$ & $377 \mathrm{~g}$ & $474 \mathrm{~g}$ & Average \\
\hline PP (uncoated) & $99 \mathrm{pF}$ & $103 \mathrm{pF}$ & $107 \mathrm{pF}$ & $110 \mathrm{pF}$ & $111 \mathrm{pF}$ & $106 \mathrm{pF}$ \\
PP (coated) & $133 \mathrm{pF}$ & $135 \mathrm{pF}$ & $139 \mathrm{pF}$ & $143 \mathrm{pF}$ & $141 \mathrm{pF}$ & $138 \mathrm{pF}$ \\
PVDF & $958 \mathrm{pF}$ & $957 \mathrm{pF}$ & $956 \mathrm{pF}$ & $957 \mathrm{pF}$ & $959 \mathrm{pF}$ & $957 \mathrm{pF}$
\end{tabular}




\section{ENERGY HARVESTING FIGURE OF MERIT COMPARISON}

To analyze the performance of a thickness-mode (33-mode) energy harvester based on a single or multiple layers of piezoelectric or electret films and a seismic mass as tested in this work, an expression for the expected power output is needed. Using the lumped parameter model as above, the power generated by the energy harvester is equal to the power dissipated in the load resistance. The magnitude of the voltage across the load resistance per unit base acceleration is obtained from Eq. (3) as

$$
\left|\frac{V}{A}\right|=\frac{R m d_{33} \omega}{\sqrt{(R C \omega)^{2}+1}}
$$

and the current through the load resistor per base acceleration is found using Ohm's law:

$$
\left|\frac{I}{A}\right|=\frac{m d_{33} \omega}{\sqrt{(R C \omega)^{2}+1}}
$$

Since the voltage across and the current through a resistor are in phase, the power generated per base acceleration squared is simply the product of Eqs. (5) and (6):

$$
\frac{P}{A^{2}}=\frac{R\left(m d_{33} \omega\right)^{2}}{(R C \omega)^{2}+1}
$$

At a given frequency, the harvested power output is maximized when the load impedance matches the output impedance of the device. For this case, the optimal load resistance is

$$
R_{\text {opt }}=\frac{1}{\omega C}
$$

Substituting this into the power expression yields

$$
\left(\frac{P}{A^{2}}\right)_{\max }=\frac{\omega m^{2} d_{33}^{2}}{2 C}
$$

which means

$$
\left(\frac{P}{A^{2}}\right)_{\max } \propto \frac{d_{33}^{2}}{\varepsilon_{33}}
$$

where $\varepsilon_{33}$ is the overall permittivity of the film (since the capacitance is $C=\varepsilon_{33} A_{e} / h$ where $A_{e}$ is the surface electrode area and $h$ is the film thickness, i.e. the electrode spacing).

Table 4. Summarized values of material selection criterion (thicknesses used to calculate permittivity are $110 \mu \mathrm{m}, 80 \mu \mathrm{m}$, and $70 \mu \mathrm{m}$ for PVDF, uncoated PP, and coated PP, respectively. Electrode area is $9.56 \mathrm{~cm}^{2}$.)

\begin{tabular}{lcccc}
\hline Sample name & $d_{33}[\mathrm{pC} / \mathrm{N}]$ & $\varepsilon_{33}[\mathrm{pF} / \mathrm{m}]$ & $d_{33}^{2} / \varepsilon_{33}\left[\mathrm{pC}^{2} \mathrm{~m} / \mathrm{N}^{2} \mathrm{~F}\right]$ & Power increase vs. PVDF [\%] \\
\hline PP (uncoated) & 13.6 & 8.86 & 20.9 & $531 \%$ \\
PP (coated) & 76.7 & 10.1 & 582 & $17500 \%$ \\
PVDF & 19.1 & 110 & 3.31 & --
\end{tabular}


According to Eq. (10), for a given base acceleration amplitude $(A)$ and under optimal electrical loading condition given by Eq. (8), as the maximum power output is proportional to the square of the piezoelectric strain constant divided by the permittivity, this ratio can be used as a criterion for choosing the best material for an energy harvester of given size. In other words, the figure of merit is $d_{33}^{2} / \varepsilon_{33}$. While the above derivation was done using simple resistive loading and a lossless harvester model, importantly, this criterion applies for more realistic conditions as well. The ratios for the three materials are summarized in Table 4. Using the parameters identified here, a seismic mass based vibration energy harvester made of PP electret film operating in the 33-mode, could produce up to 175 times the power for a given base acceleration level than a similar device made with piezoelectric PVDF due to the increased effective piezoelectric constant and decreased permittivity.

\section{CONCLUSIONS}

The transformation of vibration energy into low-power electricity has been heavily researched over the past two decades for enabling wireless and self-powered electronic components. Piezoelectric transduction is the most popular transduction mechanism for vibration-to-electricity conversion. The literature of piezoelectric energy harvesting has mainly explored brittle piezoelectric ceramics (e.g. PZTs) and limited efforts have explored PVDFs as the soft material option. This work presented figure of merit comparison of PP versus PVDF for off-resonant energy harvesting in thickness mode (33-mode) operation, revealing substantial advantage of PP over PVDF. We showed that, for thickness-mode energy harvesting scenarios (e.g. dynamic compression) at realistic ambient vibration frequencies, the figure of merit for the maximum power output is proportional to the square of the effective piezoelectric strain constant divided by the effective permittivity constant. Furthermore, we showed that PP samples yield larger effective piezoelectric constant and lower capacitance (due to voids in the material). As a consequence, under optimal electrical loading conditions and for the same volume, PP can generate more than two orders of magnitude larger electrical power as compared to PVDF due to the larger effective piezoelectric strain constant and lower permittivity of the former.

\section{ACKNOWLEDGMENT}

This work was made possible by NPRP grant No. NPRP 6-282-2-119 from the Qatar National Research Fund (A Member of The Qatar Foundation). The statements made herein are solely the responsibility of the authors.

\section{REFERENCES}

1. Beeby, S.P., M.J. Tudor, and N. White, Energy harvesting vibration sources for microsystems applications. Measurement science and technology, 2006. 17(12): p. R175.

2. $\quad$ Anton, S.R. and H.A. Sodano, A review of power harvesting using piezoelectric materials (2003-2006). Smart materials and Structures, 2007. 16(3): p. R1.

3. Cook-Chennault, K., N. Thambi, and A. Sastry, Powering MEMS portable devices-a review of nonregenerative and regenerative power supply systems with special emphasis on piezoelectric energy harvesting systems. Smart Materials and Structures, 2008. 17(4): p. 043001.

4. $\quad$ Priya, S. and D.J. Inman, Energy Harvesting Technologies. Vol. 21. 2009: Springer.

5. $\quad$ Elvin, N. and A. Erturk, Advances in Energy Harvesting Methods. 2013, New York: Springer.

6. $\quad$ Erturk, A. and D.J. Inman, Piezoelectric Energy Harvesting. 2011, Chichester: Wiley. 392 pp.

7. Shenck, N.S. and J.A. Paradiso, Energy scavenging with shoe-mounted piezoelectrics. IEEE micro, 2001(3): p. 30-42.

8. Taylor, G.W., et al., The energy harvesting eel: a small subsurface ocean/river power generator. Oceanic Engineering, IEEE Journal of, 2001. 26(4): p. 539-547. 
9. $\quad$ Granstrom, J., et al., Energy harvesting from a backpack instrumented with piezoelectric shoulder straps. Smart Materials and Structures, 2007. 16(5): p. 1810.

10. Sun, C., et al., PVDF microbelts for harvesting energy from respiration. Energy \& Environmental Science, 2011. 4(11): p. 4508-4512.

11. Anton, S., K. Farinholt, and A. Erturk, Piezoelectret foam-based vibration energy harvesting. Journal of Intelligent Material Systems and Structures, 2014: p. 1045389X14541501.

12. Gerhard-Multhaupt, R., Less can be more. Holes in polymers lead to a new paradigm of piezoelectric materials for electret transducers. Dielectrics and Electrical Insulation, IEEE Transactions on, 2002. 9(5): p. 850-859.

13. Bauer, S., R. Gerhard-Multhaupt, and G.M. Sessler, Ferroelectrets: Soft electroactive foams for transducers. Physics Today, 2004. 57(2): p. 37-44.

14. Hillenbrand, J., P. Pondrom, and G. Sessler, Electret transducer for vibration-based energy harvesting. Applied Physics Letters, 2015. 106(18): p. 183902.

15. Pondrom, P., et al., Energy harvesting with single-layer and stacked piezoelectret films. Dielectrics and Electrical Insulation, IEEE Transactions on, 2015. 22(3): p. 1470-1476.

16. Zhang, X., L. Wu, and G.M. Sessler, Energy harvesting from vibration with cross-linked polypropylene piezoelectrets. AIP Advances, 2015. 5(7): p. 077185.

17. Kornbluh, R.D., et al., From boots to buoys: promises and challenges of dielectric elastomer energy harvesting, in Electroactivity in Polymeric Materials. 2012, Springer. p. 67-93.

18. Koh, S.J.A., X. Zhao, and Z. Suo, Maximal energy that can be converted by a dielectric elastomer generator. Applied Physics Letters, 2009. 94(26): p. 262902.

19. Aureli, M., et al., Energy harvesting from base excitation of ionic polymer metal composites in fluid environments. Smart materials and Structures, 2009. 19(1): p. 015003.

20. Giacomello, A. and M. Porfiri, Underwater energy harvesting from a heavy flag hosting ionic polymer metal composites. Journal of Applied Physics, 2011. 109(8): p. 084903. 